\section{The HELIOS trial protocol: a Phase III study of ibrutinib in combination with bendamustine and rituximab in relapsed/ refractory chronic lymphocytic leukemia}

\author{
Michael Hallek', Neil E Kay², Anders Osterborg³, Asher A Chanan-Khan', \\ Michelle Mahler ${ }^{5}$, Mariya Salman ${ }^{5}$, Ying Wan ${ }^{5}$, Steven Sun ${ }^{5}$, Sen Hong \\ Zhuang $^{5}$ \& Angela Howes ${ }^{6}$
}

\begin{abstract}
Ibrutinib is an orally administered, covalent inhibitor of Bruton's tyrosine kinase with activity in B-cell malignancies based on Phase I/II studies. We describe the design and rationale for the Phase III HELIOS trial (trial registration: EudraCT No. 2012-000600-15; UTN No. U1111-1135-3745) investigating whether ibrutinib added to bendamustine and rituximab (BR) provides benefits over BR alone in patients with relapsed/refractory chronic lymphocytic leukemia/small lymphocytic lymphoma. Eligible patients must have relapsed/refractory disease measurable on CT scan and meet $\geq 1$ International Workshop on Chronic Lymphocytic Leukemia criterion for requiring treatment; patients with del(17p) are excluded. All patients receive BR (maximum six cycles) as background therapy and are randomized 1:1 to placebo or ibrutinib $420 \mathrm{mg} / \mathrm{day}$. Treatment with ibrutinib or placebo will start concomitantly with BR and continue until disease progression or unacceptable toxicity. The primary end point is progression-free survival. Secondary end points include safety, objective response rate, overall survival, rate of minimal residual disease-negative remissions, and patient-reported outcomes. Tumor response will be assessed using the International Workshop on Chronic Lymphocytic Leukemia guidelines.
\end{abstract}

Chronic lymphocytic leukemia (CLL) is the most common adult leukemia in the Western world. It is characterized by an accumulation of monoclonal mature $\mathrm{B}$ cells $\left(\mathrm{CD} 5^{+} \mathrm{CD} 23^{+}\right)$in the blood, bone marrow and secondary lymph organs $[1,2]$. Elements within the tissue microenvironment and their potential engagement through B-cell receptor (BCR) signaling have emerged as key interactive biologic players in the pathogenesis of CLL $[1,2]$. According to the WHO, small lymphocytic lymphoma (SLL) is considered a manifestation of the same underlying disorder as CLL [3].

Once diagnosed with CLL, patients often have a variable disease course, some not requiring treatment for decades and others requiring more urgent treatment [4]. Based on this clinical heterogeneity, the International Workshop on CLL (iwCLL) has defined criteria for therapeutic intervention (Box 1) [5]. The current, and most commonly used therapeutic strategy for previously untreated CLL consists of CD20-based chemoimmunotherapy combination regimens such as fludarabine, cyclophosphamide, and rituximab (FCR) [6,7]. The FCR combination has also demonstrated efficacy in patients with relapsed or refractory CLL. However, toxicity remains an important concern, especially in patients with advanced age [8]. Recently, a Phase II study evaluated the combination of bendamustine and rituximab (BR) in patients with relapsed or refractory CLL and showed important clinical activity,

'Department I of Internal Medicine \& Center of Integrated Oncology, University of Cologne, Germany

${ }^{2}$ Mayo Clinic, Rochester, MN, USA

${ }^{3}$ Department of Hematology, Karolinska University Hospital, Solna, Stockholm, Sweden

${ }^{4}$ Mayo Clinic, Jacksonville, FL, USA

${ }^{5}$ Janssen Research \& Development, Raritan, NJ, USA

${ }^{6}$ Janssen Research \& Development, High Wycombe, UK

*Author for correspondence: Tel.: +49 221 478 4401; Fax: +49 221478 5455; michael.hallek@uni-koeln.de

\section{KEYWORDS}

- B-cell receptor signaling - bendamustine $\bullet$ Bruton's tyrosine kinase $\bullet$ chronic lymphocytic leukemia - ibrutinib • rituximab - small lymphocytic lymphoma 
Box 1. International Workshop on Chronic Lymphocytic Leukemia active disease criteria indicating a need for initiating treatment for chronic lymphocytic leukemia.

1. Evidence of progressive marrow failure as manifested by the development of, or worsening of, anemia and/or thrombocytopenia

2. Massive (i.e., at least $6 \mathrm{~cm}$ below the left costal margin) or progressive or symptomatic splenomegaly

3. Massive nodes (i.e., at least $10 \mathrm{~cm}$ in longest diameter) or progressive or symptomatic lymphadenopathy

4. Progressive lymphocytosis with an increase of more than $50 \%$ over a 2 -month period or lymphocyte doubling time of less than 6 months. The doubling time can be obtained by linear regression extrapolation of absolute lymphocyte counts obtained at intervals of 2 weeks over an observation period of 2-3 months ${ }^{\dagger}$

5. Constitutional symptoms, defined as any one or more of the following disease-related symptoms or signs:

a. Unintentional weight loss of $10 \%$ or more within the previous 6 months;

b. Significant fatigue (i.e., ECOG PS score of 2 or worse; inability to work or perform usual activities);

c. Fevers higher than $100.5^{\circ} \mathrm{F}$ or $38.0^{\circ} \mathrm{C}$ for 2 or more weeks without other evidence of infection;

d. Night sweats for more than 1 month without evidence of infection.

6. Autoimmune anemia and/or thrombocytopenia that is poorly responsive to corticosteroids or other standard therapy ${ }^{\ddagger}$

${ }^{+}$In patients with initial blood lymphocyte counts of less than $30 \times 10^{9} /(30,000 / \mu \mid)$, doubling time should not be used as a single parameter to define a treatment indication. In addition, factors contributing to

lymphocytosis or lymphadenopathy other than chronic lymphocytic leukemia (e.g., infections) should be excluded.

*Not included as eligibility criteria for the HELIOS study.

ECOG PS: Eastern Cooperative Oncology Group performance status.

Data taken from [5]. kinase (BTK) that is now approved in the USA for the treatment of previously treated mantle cell lymphoma and previously treated CLL. Ibrutinib is also under development for the treatment of other B-cell malignancies [13]. BTK plays a central role in BCR signaling and B-cell development and function, and abnormalities in the BCR signaling process have been implicated in B-cell malignancy [13-15]. As such, selective BTK inhibition has been investigated as a novel approach to target malignant diseases of the hematopoietic system driven by BCR activation, such as CLL/SLL [16]. Ibrutinib binds covalently to a cysteine residue (Cys-481) in the BTK active site [17]. The covalent bond formed between ibrutinib and Cys-481 is stable, resulting in sustained inhibition of the target. For example, in cellular signal transduction assays with a B-cell lymphoma cell line, ibrutinib inhibited autophosphorylation of BTK, and phosphorylation of downstream kinases [13].

Studies with published data on the clinical activity of single-agent ibrutinib in patients with relapsed or refractory CLL or SLL include a Phase I, multicenter, dose-escalation study of ibrutinib in patients with relapsed or refractory B-cell malignancies, including CLL/SLL (PCYC-04753) [18], and a Phase Ib/II, open-label, multicenter study of ibrutinib in patients with either relapsed or refractory [19] or treatment-naive [20] CLL/SLL (PCYC-1102-CA).

In PCYC-04753, responses were seen in 11 of the 16 patients with relapsed or refractory CLL/SLL (including two with a CR) [18]. Although safety data for the patients with CLL/SLL were not reported separately, across all patients in the study, the majority of adverse events (AEs) were mild (grade 1 or 2) in severity [18]. Additionally, dose-limiting events were not observed, even with prolonged dosing, and the maximum tolerated dose of ibrutinib was not reached. The most common AEs were pain (nonspecific), respiratory symptoms (other than coughing), diarrhea, nausea/vomiting and fatigue [18].

In PCYC-1102-CA, 85 patients with relapsed or refractory CLL/SLL were enrolled into one of three treatment groups, each receiving a fixed daily dose of either 420 or $840 \mathrm{mg} /$ day of ibrutinib [19]. This relapsed/refractory population had high-risk disease with a median of four prior therapies; $65 \%$ had advanced stage disease, 33\% had $\operatorname{del}(17 \mathrm{p})$, and $36 \%$ had del(11q). Longterm therapy with ibrutinib was associated with 
modest toxicity, with most AEs being grade 1 or 2 and resolving with no delay in treatment. The most common all-grade AEs were diarrhea, fatigue, and upper respiratory tract infection, with the most common events of grade 3 or higher being neutropenia (15\%), pneumonia $(12 \%)$, and dehydration (6\%) [19]. Infections of at least grade 3 occurred more frequently early in therapy, with the exposure-adjusted rate of infections reduced by more than half after 6 months of treatment. Sustained improvement in cytopenias was also frequently observed during ibrutinib treatment (i.e., improvement occurred in approximately $80 \%$ of patients with baseline thrombocytopenia, anemia or neutropenia) [19].

In the PCYC-1102-CA study, the ORR using standard criteria $[5,21]$ was $71 \%$ in both the 420-mg cohort (two CR, 34 partial response [PR]) and the 840-mg cohort (24 PR) [19]. An additional 20 and $15 \%$ of patients in the 420 and $840 \mathrm{mg} /$ day cohorts, respectively, experienced a PR with persistent lymphocytosis. This latter response pattern represents a relatively new category and relates to the fact that some drugs that impact on BCR-mediated signaling, such as ibrutinib, may cause a transient increase in blood lymphocyte counts likely reflecting a redistribution of leukemia cells from the lymphoid tissues to the blood. Treatment-related lymphocytosis has also been seen with other agents that target BCR signaling, prompting the iwCLL to conclude that lymphocytosis is not uniformly a sign of progressive disease [21]. Indeed, very recent research has indicated that prolonged lymphocytosis during ibrutinib therapy is not associated with a suboptimal therapeutic response [22]. Intriguingly, response to ibrutinib in PCYC1102-CA did not seem to vary in relation to a number of pretreatment genomic risk factors, including the high-risk group of patients with del(17p). Overall, ibrutinib treatment resulted in a 26 -month estimated PFS rate of $75 \%$ and an OS rate of $83 \%$ [19].

In terms of dosing, pharmacokinetic analyses performed as part of the PCYC-1102-CA trial showed that the exposure to ibrutinib after treatment increased proportionally from the $420 \mathrm{mg} /$ day dose to the $840 \mathrm{mg} /$ day dose. However, no differences in ibrutinib time to peak concentration or the terminal half-life were observed between doses. Furthermore, posttreatment assessment of patient blood samples indicated full occupancy of BTK by ibrutinib for both dose levels [19].
Two additional Phase Ib/II studies have reported on the safety and efficacy of ibrutinib in combination with CD20 monoclonal antibody therapy or chemoimmunotherapy in patients with relapsed or refractory CLL/SLL. Preliminary results from one study (PCYC1109-CA) have indicated that the combination of ibrutinib ( $420 \mathrm{mg} /$ day) and ofatumumab, an anti-CD20 monoclonal antibody, is well tolerated and highly active (100\% ORR) in patients with heavily pretreated relapsed/refractory CLL/SLL ( $n=24)$ [23]. Results from the other study (PCYC-1108-CA), assessing the combination of ibrutinib (420 mg/day) plus BR in 30 patients with relapsed/refractory CLL/SLL, have suggested that this combination is also highly active with an ORR of 93\% (CR 17\%) after a median treatment duration of 16 months, a low rate of disease progression (estimated 12-month PFS was 90\%), and good tolerability [24]. Indeed, the observed safety profile for this combination was consistent with that of BR and ibrutinib monotherapy, with diarrhea, nausea, fatigue, neutropenia, and upper respiratory tract infection as the most common AEs. It is also noteworthy that early onset and relatively transient treatment-related lymphocytosis, seen in $78 \%$ of patients with ibrutinib monotherapy [19] occurred less frequently with ibrutinib plus BR [24]. Results from a Phase II, single-center, investigator-initiated clinical trial have also shown that ibrutinib in combination with rituximab was a well-tolerated regimen for patients $(n=40)$ with high-risk CLL inducing a high rate of durable remissions (ORR of 95\% [CR: 10\%]; median PFS of 78\% after 18 months) [25]. Several Phase III studies are also ongoing in patients with CLL, evaluating ibrutinib alone or as part of combination therapy in comparison to other treatment regimens.

Although there have been impressive gains in the treatment and understanding of the biology of CLL, the disease remains incurable using chemotherapy or chemoimmunotherapy [26,27]. Allogeneic stem cell transplantation may be curative, but many patients may not be suitable candidates for this type of therapy due to age, lack of donors, or other medical diseases [4]. Importantly, the more relapses patients incur, the more resistant patients become to therapy [27]. Therefore, there is a need for further development of effective and less toxic therapies for CLL. 


\section{HELIOS trial}

Herein we describe the design and rationale for the randomized, double-blind, placebocontrolled, multicenter, Phase III HELIOS study (EudraCT No. 2012-000600-15; UTN No. U1111-1135-3745), which is expected to provide additional information on treatment options available to patients with relapsed and/or refractory CLL/SLL.

\section{Objectives}

The primary objective of the HELIOS study is to determine whether the addition of ibrutinib to BR significantly improves PFS compared with $\mathrm{BR}$ alone in patients with relapsed or refractory CLL/SLL.

Secondary objectives are to:

- Evaluate the safety of ibrutinib in combination with BR;

- Evaluate the ORR (defined as the proportion of patients with a CR, a CR with incomplete marrow recovery, a PR or a nodular PR);

- Evaluate OS;

- Evaluate the rate of minimal residual disease (MRD)-negative remissions;

- Evaluate improvement in hematologic parameters;

- Evaluate improvement of disease-related symptoms;

- Evaluate patient-reported outcomes as measured by the European Organization for Research and Treatment of Cancer Quality of Life Questionnaire (EORTC-QLQ)-C30, EORTC QLQ-CLL 16, EQ-5D-5L and Functional Assessment of Chronic Illness Therapy (FACIT)-Fatigue Scale;

- Characterize the pharmacokinetics (PK) of ibrutinib and explore its potential effect on BR $\mathrm{PK}$ and the potential relationships between ibrutinib exposure and relevant clinical or biomarker information;

- Examine biomarkers related to BCR and compensatory signaling pathways and explore their association with resistance to ibrutinib treatment.

\section{Key eligibility criteria}

Eligible patients must be aged 18 years or older with a diagnosis of CLL or SLL that meets published diagnostic criteria [5], and must also have relapsed or refractory disease following at least one prior line of systemic therapy consisting of at least two cycles of a chemotherapy-containing regimen. Prior CD20 monoclonal antibody therapy was not a requirement for inclusion. Patients must have active disease meeting at least one of the iwCLL 2008 criteria for initiating treatment (criteria 1-5 in Box 1). Patients enrolled in the study must also have measurable nodal disease by computed tomography (CT) of the neck, chest, abdomen, and pelvis, an Eastern Cooperative Oncology Group performance status score of 0 or 1 , and hematologic and biochemical parameters within prespecified limits. All eligible patients (or their legally acceptable representatives) must provide written informed consent.

Exclusion criteria include any anticancer therapy in the 3-10 weeks prior to randomization (depending on the agent) and/or major surgery within 4 weeks prior to randomization, prior treatment with ibrutinib or any other BTK inhibitor, $\operatorname{del}(17 \mathrm{p})$ as defined as the presence of $\operatorname{del}(17 \mathrm{p})$ in $\geq 20 \%$ of cells examined by FISH, nonresponse to a previous bendamustine-containing regimen or relapse within 24 months, prior hematopoietic stem cell transplant or a goal of therapy of tumor debulking prior to stem cell transplant, or a history of prior malignancy (except that treated with curative intent $\geq 3$ years previously, adequately treated nonmelanoma skin cancer, or cervical cancer in situ without evidence of disease).

\section{Study design \& treatment plan}

Upon meeting the eligibility criteria, patients will be randomized in a 1:1 ratio to receive either placebo (arm A; placebo -3 capsules) or ibrutinib (arm B; ibrutinib $420 \mathrm{mg}-3 \times 140 \mathrm{mg}$ capsules) according to the planned schedule (Figure 1). All patients will receive therapy with BR for a maximum of six cycles. Bendamustine hydrochloride will be administered as $70 \mathrm{mg} / \mathrm{m}^{2}$ infused intravenously (IV) over $30 \mathrm{~min}$. Rituximab will be administered as $375 \mathrm{mg} / \mathrm{m}^{2}$. A cycle will be defined as 28 days. Study medication will be self-administered orally once daily on a continuous schedule until disease progression or unacceptable toxicity.

Randomization will be balanced by using randomly permuted blocks and will be stratified. Stratification factors will include whether the patient is refractory to purine analog therapy (yes or no) and number of prior lines of therapy 


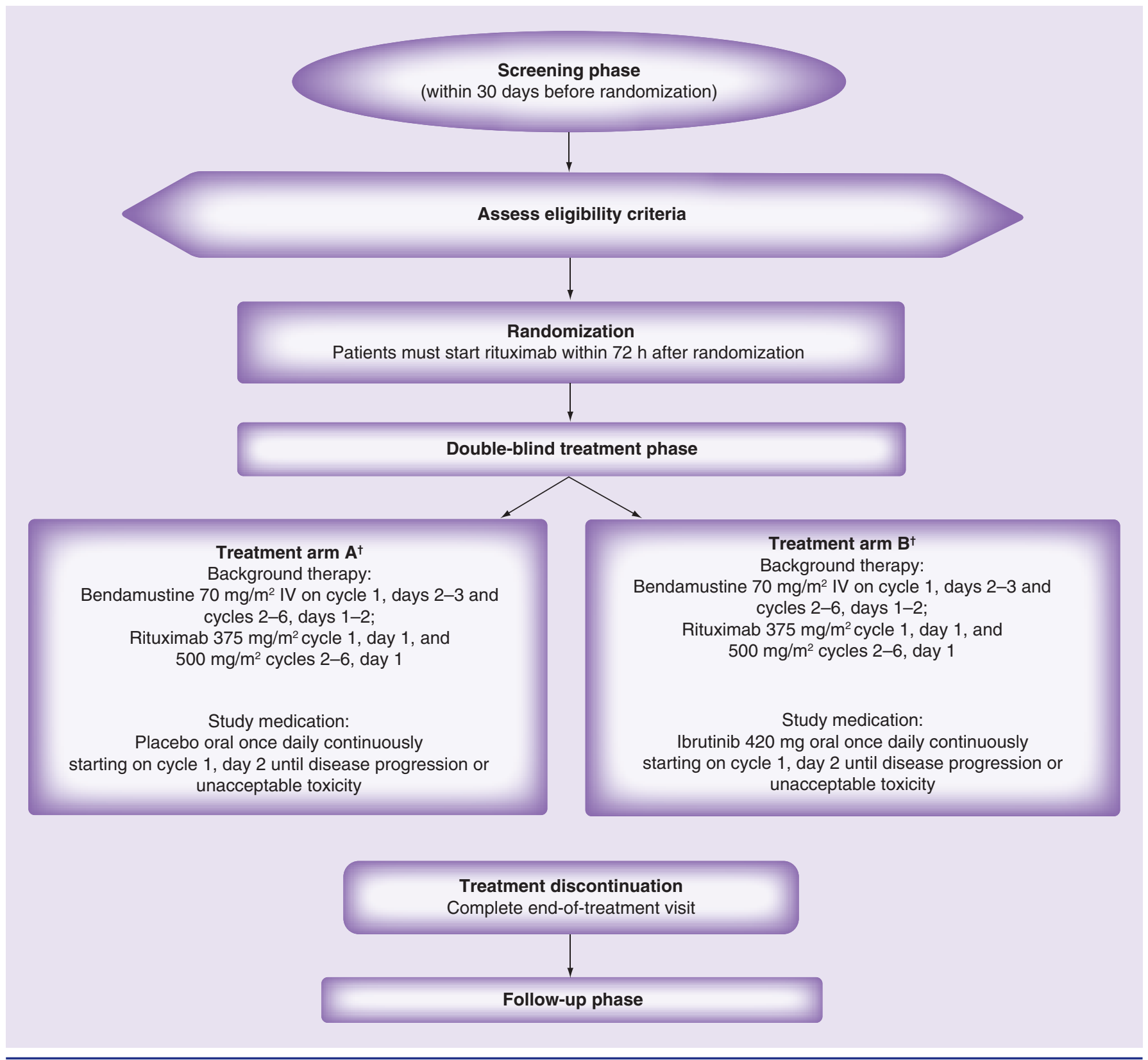

Figure 1. Study design schema.

${ }^{\dagger}$ A cycle will be defined as 28 days, except for cycle 1, which will be 29 days.

IV: Intravenous.

( 1 or $>1)$. For stratification, refractory is defined specifically as a failure to respond (i.e., progressive disease or stable disease) to a purine analog or a recurrence of disease within 12 months of the last dose of purine analog therapy.

The study will consist of a screening phase, a treatment phase, and a follow-up phase (Figure 1). The treatment phase will extend from randomization until study drug discontinuation. Patients will receive both $\mathrm{BR}$ and oral study medication for the first six cycles, and thereafter will receive oral study medication until disease progression or unacceptable toxicity. For patients who discontinue BR prior to completing six cycles, treatment with oral study medication will continue. The follow-up phase will begin once a patient discontinues study medication and will continue until death, loss to follow-up, consent withdrawal, or study end, whichever occurs first. Data will be collected either in person if 
the patient is on-site or via a telephone call with the patient by the investigator staff. Trial study end is defined as the point at which either $80 \%$ of the patients have died or 4 years after the last patient is randomized into the study, whichever occurs first.

Blinded treatment doctrine will be followed in order to reduce potential bias during data collection and evaluation of end points. Both the patient and the investigator will be blinded to treatment allocation.

\section{Efficacy evaluations}

Assessment of tumor response and progression will be conducted in accordance with iwCLL guidelines [5] with the modification that treatment-related lymphocytosis is not considered progression according to clarification of the iwCLL criteria [21]. The investigator will evaluate sites of disease by radiological imaging using CT scans with intravenous contrast of the neck, chest, abdomen and pelvis (MRI will be used to evaluate sites of disease that cannot be adequately imaged using CT), physical examination, or other procedures as necessary, and review of hematology and clinical chemistry results. A central laboratory will perform complete blood count testing. Peripheral blood or bone marrow aspirate/biopsy with flow cytometry assessment(s) for MRD will be done via a central laboratory if there is evidence of CR by clinical parameters. There will be central review of response and progression by Independent Review Committee (IRC). The primary efficacy analysis of PFS will be based on assessment by the IRC. Patient-reported symptoms, functional status, and well-being will also be measured.

\section{PK evaluations}

In both treatment arms, PK samples will be collected from all patients on day 2 during cycles 1 and 2 and evaluated for ibrutinib levels. In addition, for a subset of patients at selected sites, sampling will be performed in both treatment arms to explore whether ibrutinib has any effect on the PK of BR.

\section{Biomarker evaluations}

Blood samples will be collected and analyzed to better understand the mechanism of action of ibrutinib, with an aim to investigate predictors of resistance. Plasma protein analysis may include evaluation of IL-4, IL-6 and CXCL-12. Isolated peripheral B-lymphocytes may also be evaluated for protein expression of ZAP-70, CD38, RNA/DNA status for IGVH (mutated or non-mutated) and other RNA/DNA signatures that may predict acquired resistance. Analyses will be performed within each treatment group in total and stratified by clinical covariates or molecular subgroups. The associations of biomarkers with clinical response or time-to-event end points will be assessed using appropriate statistical methods. Biomarker analysis may be deferred or not performed if during or at the end of the study it becomes clear that the analysis will not have sufficient scientific value for biomarker evaluation, or if there are not enough samples or responders to allow for adequate biomarker evaluation.

\section{Safety evaluations}

During the study, safety evaluations will include AE monitoring, physical examinations, concomitant medication usage and clinical laboratory parameters (hematology, chemistry, coagulation). All AEs (with the exception of progression of CLL) and special reporting situations, whether serious or nonserious, will be reported from the time a signed and dated informed consent form is obtained until 30 days following the last dose of study drug or until the start of a subsequent systemic antineoplastic therapy, if earlier. AEs reported after 30 days following the last dose of study drug will also be reported if considered related to study drug. AEs will be coded using the Medical Dictionary for Regulatory Activities; the severity of nonhematologic AEs will be assessed using National Cancer InstituteCommon Terminology Criteria for Adverse Events Version 4.03, and severity of hematologic AEs will be assessed using iwCLL 2008 criteria.

\section{Statistical rationale \\ - Analysis sets}

The intent-to-treat population is defined as all patients randomized into the study, and will be classified according to assigned treatment group, regardless of the actual treatment received. This population will be used for primary efficacy analyses, including PFS and OS, and all analyses of disposition, demographic and baseline disease characteristics.

The safety population is defined as all randomized patients who receive at least one dose of study medication. This population will be used for all safety analyses and all analyses of exposure and treatment compliance. All data will be 
analyzed according to the treatment patients actually received. The PK evaluable population will include all patients who receive at least one dose of study medication and have at least one post-treatment PK sample.

\section{- Sample size}

Approximately 580 patients (290 per treatment group) will be randomized in a 1:1 fashion. The study is designed to detect a hazard ratio of 0.7 for the ibrutinib plus BR group relative to the placebo plus BR group, with $90 \%$ power at a one-sided significance level of 0.025 , using a group sequential testing design.

\section{- Analysis methods}

The primary efficacy analysis will be based on the PFS determined by the IRC and will be performed on the intention-to-treat population. The Kaplan-Meier method will be used to estimate the distribution of PFS for each treatment group. The stratified log-rank test will be used to compare the PFS between the two treatment groups. The median PFS will be provided for each treatment group and hazard ratios and associated $95 \%$ CI will be calculated.

For the secondary efficacy end points, OS will be compared using the stratified log-rank test. The Kaplan-Meier method will be used to estimate the distribution of OS for each treatment group. The ORR and the rate of MRD-negative remissions will be obtained and comparison of the rates will be performed between the two treatment groups using the Cochran-Mantel-Haenszel $\chi^{2}$ test. Disease-related symptom improvement and hematologic improvement data will be summarized as appropriate. Scores from EORTC QLQ-C30, EORTC QLQ-CLL 16, EQ-5D-5L and the FACIT-Fatigue will be descriptively summarized by treatment group.

The plasma concentration data for ibrutinib (and, if possible and judged relevant to the performed PK analyses, the metabolite PCI-45227), as well as for BR will be summarized at each time point using descriptive statistics. Population PK analysis of ibrutinib and BR plasma concentration-time data will be performed using nonlinear mixed-effects modeling. Available patient characteristics will be tested as potential covariates affecting PK parameters. Model-derived exposure parameters may be subjected to further explore PK/pharmacodynamic correlation between exposure and relevant clinical or biomarker information.
Biomarker analyses will be performed within each treatment group in total and stratified by clinical covariates or molecular subgroups. The associations of biomarkers with clinical response or time-to-event end points will be assessed using the appropriate statistical methods.

Safety variables will be tabulated by descriptive statistics (n, mean, median, standard deviation, minimum and maximum; or $\mathrm{n}$ and percent). No formal statistical testing of the safety variables is planned.

\section{- Interim analysis}

An interim analysis using classical O'BrienFleming boundary for both efficacy and futility will be conducted after observing approximately $50 \%$ PFS events [28]. In addition to the planned interim analysis, the Data Monitoring Committee will meet at appropriate and periodic intervals to review the cumulative safety data throughout the study.

\section{Future perspective}

Ibrutinib has shown promising clinical activity as a single agent for the treatment of several B-cell malignancies, including relapsed and refractory CLL/SLL [18,19]. Moreover, early results from a Phase Ib/II study of ibrutinib plus BR in 30 patients with relapsed or refractory CLL/SLL have suggested that the combination may provide additional efficacy benefits [24]. The HELIOS trial will further analyze whether the addition of ibrutinib to standard BR therapy will provide prolonged disease control compared with $\mathrm{BR}$ alone in this relatively high risk patient population. It will also be of interest to observe any effect on ORR, including CR rate and the rates of MRD-negative disease. This trial will be an important large-scale randomized study in the evolution of combination treatments for relapsed/refractory CLL/SLL and will provide additional robust sets of information/data on the efficacy and tolerability of ibrutinib plus BR in this setting. Indeed, it is hoped that the outcomes of the HELIOS trial, and other ongoing studies, will lead to the availability of additional treatment options for heavily pretreated patients with relapsed or refractory CLL.

Financial \& competing interests disclosure

A Osterborg was on the steering committee for this study and has received honoraria for scientific lectures and ad boards for Janssen Research \& Development. A Osterborg has nothing to disclose with regard to Pharmacyclics. 


\begin{abstract}
A Howes, $M$ Mahler, M Salman, S Sun, Y Wan and SH Zhuang are employees of Janssen Research \& Development (Pharmaceutical companies of Johnson \& Johnson). A Howes, S Sun, Y Wan, and SH Zhuang also have stock ownership or options in Johnson \& Johnson to disclose. A Chanan-Khan and N Kay do not have any conficts to disclose with regard to this study. The authors have no other relevant affiliations or financial involvement with any organization or entity with a financial interest in or financial conflict with the subject matter or materials
\end{abstract}

discussed in the manuscript apart from those disclosed.

Medical writing assistance was provided by $R$ Daniel and M Mehta (PPSI, a PAREXEL company) and was funded by Janssen Research \& Development.

\section{Open Access}

This work is licensed under the Creative Commons Attribution-NonCommercial 3.0 Unported License. To view a copy of this license, visit http://creativecommons.org/ licenses/by-nc-nd/3.0/

\section{EXECUTIVE SUMMARY}

\section{Ibrutinib}

- Ibrutinib (PCI-32765) is a first-in-class, orally administered, potent, covalent inhibitor of Bruton's tyrosine kinase that has shown interesting clinical activity as a single agent for the treatment of several B-cell malignancies, including chronic lymphocytic leukemia (CLL)/small lymphocytic lymphoma (SLL).

\section{HELIOS study}

- A large-scale, randomized, double-blind, placebo-controlled, multicenter Phase III study designed to determine the benefits and risks of combining ibrutinib and bendamustine and rituximab (BR) in patients with relapsed or refractory $\mathrm{CLL} / \mathrm{SLL}$ following at least one line of prior systemic therapy.

- All patients will receive background therapy of BR for a maximum of six cycles and will be randomized 1:1 to receive either placebo or ibrutinib at a daily dose of $420 \mathrm{mg}$.

- Approximately 580 patients (290 per treatment group) will be randomized to observe 342 progression-free survival (PFS) events.

\section{Objectives}

- The primary objective of the HELIOS study is to determine whether the addition of ibrutinib to BR significantly improves PFS compared with BR in patients with relapsed or refractory CLL/SLL. Secondary objectives include determination of safety, objective response rate, overall survival, rate of minimal residual disease-negative remissions, improvement in hematologic parameters, improvement in disease-related symptoms, improvement in patientreported outcomes, and changes in pharmacokinetic parameters and biomarkers related to B-cell receptor and compensatory signaling pathways.

\section{Statistical methods}

- The primary efficacy end point of PFS will be determined by an Independent Review Committee. The study is designed to detect a hazard ratio of 0.7 for the ibrutinib plus BR group relative to the placebo plus BR group with $90 \%$ power at a one-sided significance level of 0.025 , using a group sequential testing design.

- Two analyses are planned for the HELIOS trial: an interim analysis using an O'Brien-Fleming boundary, and a final analysis. An independent Data Monitoring Committee will review safety data periodically and data from the interim analysis.

\section{References}

Papers of special note have been highlighted as: - of interest; $\bullet$ of considerable interest

1 Burger JA. Inhibiting B-cell receptor signaling pathways in chronic lymphocytic leukemia. Curr. Hematol. Malig. Rep. 7(1), 26-33 (2012).

- Discussed B-cell receptor signaling pathways in chronic lymphocytic leukemia (CLL). It provides an overview of $\mathrm{B}$-cell receptor- associated kinases and their therapeutic promise in CLL.

2 Hallek M. Signaling the end of chronic lymphocytic leukemia: new frontline. treatment strategies. Blood 122(23), 3723-3734 (2013).

- Summarizes the currently available diagnostic and therapeutic tools for CLL and gives an integrated recommendation of how to manage the disease. It also discusses how novel agents for CLL might be integrated into sequential treatment approaches in the future.

3 Campo E, Swerdlow SH, Harris NL, Pileri S, Stein H, Jaffe ES. The 2008 WHO classification of lymphoid neoplasms and beyond: evolving concepts and practical applications. Blood 117(19), 5019-5032 (2011).

4 Gribben JG, O'Brien S. Update on therapy of chronic lymphocytic leukemia. J. Clin. Oncol. 29(5), 544-550 (2011). 
5 Hallek M, Cheson BD, Catovsky D et al. Guidelines for the diagnosis and treatment of chronic lymphocytic leukemia: a report from the International Workshop on Chronic Lymphocytic Leukemia updating the National Cancer Institute-Working Group 1996 guidelines. Blood 111(12), 5446-5456 (2008).

6 Rai KR, Peterson BL, Appelbaum FR et al. Fludarabine compared with chlorambucil as primary therapy for chronic lymphocytic leukemia. N. Engl. J. Med. 343(24), 1750-1757 (2000).

7 Hallek M, Fischer K, Fingerle-Rowson G et al. Addition of rituximab to fludarabine and cyclophosphamide in patients with chronic lymphocytic leukaemia: a randomised, open-label, Phase 3 trial. Lancet 376(9747), 1164-1174 (2010).

8 Badoux XC, Keating MJ, Wang X et al. Fludarabine, cyclophosphamide, and rituximab chemoimmunotherapy is highly effective treatment for relapsed patients with CLL. Blood 117(11), 3016-3024 (2011).

9 Fischer K, Cramer P, Busch R et al. Bendamustine combined with rituximab in patients with relapsed and/or refractory chronic lymphocytic leukemia: a multicenter Phase II trial of the German Chronic Lymphocytic Leukemia Study Group. J. Clin. Oncol.29(26), 3559-3566 (2011).

- Reports on the combination of bendamustine and rituximab (BR) in patients with relapsed and/or refractory CLL in a Phase II clinical trial. BR treatment demonstrated promising efficacy and safety results with a complete response rate of $9 \%$, an overall response rate (ORR) of $59 \%$, median progression-free survival of 15 months, and overall survival of 34 months

10 Zaja F, Mian M, Volpetti S et al. Bendamustine in chronic lymphocytic leukemia: outcome according to different clinical and biological prognostic factors in the everyday clinical practice. Am. J. Hematol. 88(11), 955-960 (2013).

11 Eichhorst B, Dreyling M, Robak T, Montserrat E, Hallek M. Chronic lymphocytic leukemia: ESMO Clinical Practice Guidelines for diagnosis, treatment and follow-up. Ann. Oncol. 22(Suppl. 6), vi50-vi54 (2011).

12 National Comprehensive Cancer Network. NCCN Clinical Practice Guidelines in Oncology: Non-Hodgkin's Lymphomas Version I. 2013. National Comprehensive
Cancer Network Website.

www.nccn.org

13 Honigberg LA, Smith AM, Sirisawad M et al. The Bruton tyrosine kinase inhibitor PCI-32765 blocks B-cell activation and is efficacious in modes of autoimmune disease and B-cell malignancy. Proc. Natl Acad. Sci. USA. 107(29), 13075-13080 (2010).

14 Khan WN. Regulation of B lymphocyte development and activation by Bruton's tyrosine kinase. Immunol. Res. 23(2-3), 147-156 (2001).

15 Wiestner A. Targeting B-cell receptor signaling for anticancer therapy: the Bruton's tyrosine kinase inhibitor ibrutinib induces impressive responses in B-cell malignancies. J. Clin. Oncol. 31(1), 128-130 (2013).

16 de Rooij MFM, Kuil A, Geest C et al. The clinically active BTK inhibitor PCI-32765 targets B-cell receptor-and chemokinecontrolled adhesion and migration in chronic lymphocytic leukemia. Blood 119(11), 2590-2594 (2012).

17 Pan Z, Scheerens H, Li SJ et al. Discovery of selective irreversible inhibitors for Bruton's tyrosine kinase. Chem. Med. Chem. 2(1), 58-61 (2007)

18 Advani RH, Buggy JJ, Sharman JP et al. Bruton tyrosine kinase inhibitor ibrutinib (PCI-32765) has significant activity in patients with relapsed/refractory B-cell malignancies. J. Clin. Oncol. 31(1), 88-94 (2012).

- Shows data from a Phase I trial of singleagent ibrutinib in patients with relapsed or refractory B-cell malignancies. The study demonstrated that orally administered ibrutinib was well tolerated and induced responses in the majority of patients with CLL/small lymphocytic lymphoma.

19 Byrd JC, Furman RR, Coutre SE et al. Targeting BTK with ibrutinib in relapsed chronic lymphocytic leukemia. N. Engl. J. Med. 369(1), 32-42 (2013).

-• Reports on data from a Phase Ib/II trial of ibrutinib in patients with CLL/small lymphocytic lymphoma. The study demonstrated that long-term therapy with ibrutinib was associated with modest toxicity, an ORR of $71 \%$, progression-free survival rate of $75 \%$, and an overall survival rate of $83 \%$.

20 O'Brien S, Furman RR, Coutre SE et al. Ibrutinib as initial therapy for elderly patients with chronic lymphocytic leukaemia or small lymphocytic lymphoma: an open-label, multicentre, Phase $1 \mathrm{~b} / 2$ trial. Lancet Oncol. 15(1), 48-58 (2013).

21 Hallek M, Cheson BD, Catovsky D et al. Response assessment in chronic lymphocytic leukemia treated with novel agents causing an increase of peripheral blood lymphocytes. Blood (2012).

http://bloodjournal.hematologylibrary.org

22 Woyach JA, Smucker K, Smith LL et al. Prolonged lymphocytosis during ibrutinib therapy is associated with distinct molecular characteristics and does not indicate a suboptimal response to therapy. Blood 123(12), 1810-1817 (2014).

23 Jaglowski SM, Jones JA, Flynn JM et al. A Phase Ib/II study evaluating activity and tolerability of BTK inhibitor PCI-32765 and ofatumumab in patients with chronic lymphocytic leukemia/small lymphocytic lymphoma (CLL/SLL) and related diseases. J. Clin. Oncol. 30 (Suppl.) Abstract 6508, (2012).

24 Brown JR, Barrientos JC, Barr PM et al. Ibrutinib in combination with bendamustine and rituximab is active and tolerable in patients with relapsed/refractory CLL/SLL: final results of a Phase 1b study. Blood 122(21), Abstract 525 (2013).

- Final study results demonstrate that the combination of ibrutinib and BR is highly active with an ORR of $93 \%$, low rate of disease progression and good tolerability.

25 Burger JA, Keating MJ, Wierda WG et al. Ibrutinib in combination with rituximab (iR) is well tolerated and induces a high rate of durable remissions in patients with high-risk chronic lymphocytic leukemia (CLL): new, updated results of a Phase II trial in 40 patients. Blood 122(21), Abstract 675 (2013).

- Reports on data from a Phase II trial of ibrutinib in combination with rituximab in high-risk CLL patients. The study shows that this combination of ibrutinib plus rituximab was well tolerated and induced a high rate of durable remissions.

26 Hillmen P. Using the biology of chronic lymphocytic leukemia to choose treatment. Hematology Am. Soc. Hematol. Educ. Program 2011, 104-109 (2011).

27 Maddocks KJ, Lin TS. Update in the management of chronic lymphocytic leukemia. J. Hematol. Oncol. 2(29), 1-8 (2009).

28 O’Brien PC, Fleming TR. A multiple testing procedure for clinical trials. Biometrics 35(3), 549-556 (1979). 